\title{
Um Ambiente Social Virtual de Auxílio à Aprendizagem Informal na Área de Saúde
}

\author{
Bruno Elvis Costa Rodrigues da Silva ${ }^{1}$, Francisco Milton Mendes Neto ${ }^{1}$, Cicilia \\ Raquel Maia Leite ${ }^{1}$, Jerffeson Gomes Dutra ${ }^{2}$
}

${ }^{1}$ Programa de Pós-Graduação em Ciência da Computação - Universidade do Estado do Rio Grande do Norte (UERN) e Universidade Federal Rural do Semi-Árido (UFERSA) $-\mathrm{RN}$, Brasil.

${ }^{2}$ Laboratório de Engenharia de Software - Universidade Federal Rural do Semi-Árido (UFERSA) CEP 59.625-900 - Mossoró - RN - Brasil

\{elvisbruno123, jerffesongd, ciciliamaia, miltonmendes\}@gmail.com

\begin{abstract}
Informal learning contributes to the acquisition of knowledge in various fields and in the health is no different. When patients have access to information about their health status, becomes active agents in the treatment of their disease. In view of the reality that is the occurrence of chronic diseases in the population currently, it is necessary that the patient has access to contents that contribute positively to the treatment. Thus, this work proposed the development of an environment where chronic diseases patients will have access to information about their diseases based on their profile and the reality in which they are inserted.
\end{abstract}

Resumo. A aprendizagem informal contribui para a aquisição do conhecimento em diversas áreas e na saúde não é diferente. Quando pacientes tem acesso a informações a respeito do estado de saúde deles, se tornam agentes ativos no tratamento da sua doença. Diante da realidade em que se encontra a ocorrência de doenças crônicas na população atualmente, é necessário que o paciente tenha acesso a conteúdos que contribuam de forma positiva para o seu tratamento. Dessa forma, este trabalho propõe o desenvolvimento de um ambiente onde pacientes de doenças crônicas tenham acesso a informações a respeito de suas doenças com base no seu perfil e na realidade em que estão inseridos.

\section{Introdução}

Quando o conhecimento é adquirido pela experiência diária do usuário e pelo compartilhamento de informações em suas relações sociais, acontece a chamada aprendizagem informal [Jiugen et al. 2011]. Esse tipo de aquisição do conhecimento acontece dentro de diversas áreas, e a saúde apresenta um grande potencial de aplicação.

Segundo IBGE (2009), um dos fatores que influenciam a não adesão dos portadores de doenças crônicas ao tratamento médico é a falta de conhecimento acerca da sua doença e da sua condição de saúde. Moreira (2015) afirma que a educação em saúde constitui um importante recurso dos indivíduos para a determinação do seu bemestar físico, psíquico e social. De acordo com o Health Promotion Glossary [WHO 
2008], publicado pela Organização Mundial de Saúde (OMS), a educação em saúde é o conjunto de competências cognitivas e sociais que determinam a capacidade dos indivíduos para compreender e usar informações de modo que promovam e mantenham uma boa saúde.

No ano de 2014, um levantamento do Ministério da Saúde, em parceria com o Instituto Brasileiro de Geografia e Estatística (IBGE), afirmou que cerca de $40 \%$ da população brasileira adulta, o equivalente a 57,4 milhões de pessoas, possui pelo menos uma doença crônica não transmissível.

As doenças crônicas não transmissíveis são responsáveis por mais de $72 \%$ das causas de mortes no Brasil. A hipertensão arterial, o diabetes, a doença crônica de coluna, o colesterol e a depressão são as que apresentam maior prevalência no país [Portal Brasil 2014].

O diabetes apresenta algumas características que o torna especial entre as demais doenças crônicas, visto que todos os pacientes diabéticos têm necessidades diferentes. Isto acontece devido às diferenças relacionadas com a idade e comorbidades, motivação, rotinas diárias etc [Fioravanti et al. 2011].

O uso de Tecnologias da Informação e Comunicação (TICs) modernas e corretamente projetadas pode reduzir os índices de erros clínicos, melhorar o suporte dos cuidados em saúde, aumentar a eficiência no tratamento e melhorar a qualidade de vida do paciente [Montiel et al. 2015].

Diante deste cenário, a aprendizagem informal serve como um meio de aquisição do conhecimento a respeito da saúde do próprio paciente, não sendo necessário que ele esteja inserido no ambiente hospitalar. Este conceito, aliado às TICs, remete à chamada Saúde 2.0, que corresponde ao estímulo do indivíduo a ser responsável pelos cuidados relativos à sua própria saúde através do uso das ferramentas da Web 2.0, que são as redes sociais, registros pessoais de saúde, serviços etc.

No contexto da Saúde 2.0, foi desenvolvido o MobiLEHealth [Mendes Neto et al. 2014], um ambiente de aprendizagem ubíqua voltado para pacientes com doenças crônicas. Este ambiente, por meio do uso de dispositivos móveis, é capaz de adequar-se às características particulares dos usuários, fornecendo conteúdo de acordo com as suas necessidades de saúde.

Dessa forma, este trabalho aborda o desenvolvimento de uma rede social semântica que, ligada ao MobiLEHealth, servirá como um ambiente de troca de experiências entre pacientes com doenças crônicas e acesso a informações relacionadas à doença e seu estado de saúde, sendo, inicialmente, aplicado aos diabéticos. Tudo isso levando em conta o perfil do usuário e sua realidade de vivência com a doença. $O$ sistema aqui abordado funciona como uma ferramenta que será integrada ao sistema desenvolvido por Mendes Neto et al. (2014), Sombra (2015), Moreira (2015) e Costa (2015).

\section{Aprendizagem Informal}

A aprendizagem informal é o processo contínuo de aquisição do conhecimento por um indivíduo. Sendo este responsável pelo seu aprendizado e desenvolvimento, que ocorre através de suas experiências cotidianas [Wang e Shen 2012]. Moreira (2015) afirma que 
esse tipo de aprendizagem está relacionado com a busca por novos conhecimentos e habilidades, de modo não planejado ou estruturado. De forma consciente ou inconsciente, ela emerge a partir de alguma demanda ou necessidade.

Jiugen, Ruonan e Xiaoqiang (2011) destacam as principais características da aprendizagem informal:

- Autonomia: para que a aprendizagem aconteça, o indivíduo deve ser responsável por ela, determinando o objetivo a ser alcançado, o conteúdo a ser abordado e como este será processado;

- Conhecimento: diz respeito ao que é obtido por meio de interações sociais e profissionais;

- Diversidade: onde a aprendizagem é obtida a partir das experiências diárias do indivíduo, recursos do ambiente, bibliotecas, redes sociais, entre outros.

A aprendizagem, quando ocorre em ambientes informais, relaciona-se essencialmente com aprendizagens sociais, entrelaçando-se com a vida dos indivíduos de acordo com as suas experiências. Devido a isso, o contexto diário do indivíduo contribui fortemente para sua aprendizagem, visto que ela pode acontecer através de atividades diárias, interações sociais, por iniciativa própria, pela reflexão e pelos próprios erros [Castleton, Gerber e Pillay 2006].

Ambientes que proporcionam a aprendizagem informal na área da saúde favorecem a disseminação do conhecimento a respeito das condições de saúde dos envolvidos. Atrelado à experiência e acompanhamento de um profissional, a aquisição destas informações pode contribuir positivamente para a qualidade de vida de um determinado indivíduo, uma vez que, a partir do momento em que toma conhecimento a respeito da sua doença e o tratamento inerente a ela, este paciente tem maiores chances de tratar ou erradicar a sua patologia.

Outro aspecto importante com relação à inserção de aprendizagem informal no âmbito da saúde é o protagonismo do paciente em questões relacionadas à sua saúde, visto que, com maior conhecimento, ele poderá conviver melhor com a sua doença, evitar futuros problemas, trocar informações com outros indivíduos que também estejam na mesma situação, entre outras possibilidades.

\section{Saúde 2.0}

Com a Web 2.0, a internet se tornou algo presente no convívio social das pessoas. Através das suas ferramentas é possível o compartilhamento constante de informações e experiências, além da facilidade no acontecimento de interações sociais, que podem ser feitas a qualquer momento, independentemente do local e da distância em que os indivíduos se encontram.

Com a evolução das ferramentas da Web 2.0, diversos contextos foram contemplados com as facilidades que ela oferece. No contexto da saúde não é diferente, onde, através desses meios, o indivíduo se torna um agente ativo na busca de conhecimento a respeito da sua saúde. A esta prática dá-se o nome de Saúde 2.0.

Jacopetti (2011) define a Saúde 2.0 como o uso de software social e sua capacidade de promover a colaboração entre pacientes, seus médicos e outros profissionais da área da saúde. 
Santos (2008) destaca que através das redes sociais, blogs, compartilhamento de vídeos, fotos e ideias, as pessoas não precisam e nem devem mais ficar desinformadas ou vítimas de profissionais desqualificados, ou seja, a grande arma da Saúde 2.0 é a boa informação. Com o advento da Saúde 2.0 muda-se o modo como os pacientes se relacionam e compartilham suas informações e conhecimentos. As pessoas que apresentam algum sintoma, inicialmente, buscam informações a respeito do seu possível problema e só depois buscam a ajuda de algum profissional. Neste processo inclui-se a união da aprendizagem informal e das tecnologias e ferramentas da Web 2.0, voltados à construção coletiva do conhecimento na saúde [Fernandez-Luque et al. 2010].

Dessa forma, o paciente se torna um elemento ativo no processo de tratamento e conhecimento da sua doença, que, associado ao conhecimento do profissional de saúde, pode garantir que o controle e/ou cura da sua doença seja feita de forma mais rápida e eficiente.

\section{Redes Sociais Semânticas}

O fato de possuir uma grande quantidade de dados a respeito dos mais variados assuntos, faz com que a internet seja um repositório de informações em larga escala, oferecendo assim, a qualquer indivíduo, várias alternativas de acesso à informação. Por outro lado, essa característica faz com o que acesso a determinados dados seja difícil, visto que nem sempre o usuário terá acesso à informação que ele realmente necessita.

De acordo com Chenu-Abente, Giunchiglia e Cernuzzi (2015), as redes sociais on-line oferecem serviços que permitem aos usuários a gerência de suas relações sociais, de modo que seja possível compartilhar conteúdos próprios ou de terceiros, ter acesso aos conteúdos partilhados por outros usuários, trocar informações, mensagens etc. O que gera uma grande quantidade de dados, que ficam disponíveis a praticamente todos os participantes desta rede.

No intuito de fazer com que um usuário tenha acesso a determinada informação que é importante para ele, mesmo que seu círculo de amigos não apresente este mesmo interesse, busca-se inserir os conceitos e ferramentas da Web Semântica nas redes sociais.

Paydar, Kahani e Zarrinkalam (2013) afirmam que a Web Semântica tem como missão tornar a web um ambiente em que não só as pessoas, mas também as máquinas possam entender a semântica dos conteúdos publicados. Segundo Kinsella et al. (2008), o objetivo da Web Semântica é fornecer as ferramentas necessárias para definir as normas extensíveis e flexíveis para a troca de informações. Dessa forma, as Redes Sociais Semânticas consistem em redes sociais que possuem as técnicas e ferramentas oferecidas pela Web Semântica, podendo assim se adaptar de forma semântica de acordo com os interesses individuais de cada usuário, possibilitando o acesso a informações cada vez mais relevantes para aqueles que as buscam.

Para o desenvolvimento de uma rede social desse tipo, utiliza-se ontologias com diversas características, cada uma orientada ao interesse da rede. Um tipo de metodologia utilizada é a Friend-of-a-Friend (FOAF), que, segundo Pankong, Prakancharoen e Buranarach (2012), funciona como um vocabulário para descrever os perfis dos usuários, seus relacionamentos e suas atividades. O FOAF pode ser usado 
para integrar os dados presentes em uma rede social, permitindo que a informação extraída seja descrita de maneira uniforme, incluindo o perfil do usuário (contas de usuário, nome, sobrenome, amigos etc.), listas, grupos que ele participa e eventos do seu interesse.

\section{Trabalhos Relacionados}

Este trabalho trata de uma Rede Social Semântica voltada para pacientes diabéticos, de modo que possa favorecer a aprendizagem informal destes indivíduos no contexto da saúde. Dessa forma, foram estudados alguns trabalhos que propuseram ideias relacionadas à proposta deste trabalho, bem como seu desenvolvimento e solução.

Weitzel e Oliveira (2010) realizaram um estudo sobre o desenvolvimento de um sistema de recomendação de informação em saúde baseado no perfil do usuário. $O$ trabalho aborda a contextualização da necessidade de sistemas de recomendação para entregar ao usuário informações relevantes com base no seu perfil.

Lee et al. (2012) propõem uma abordagem para desenvolver uma rede social semântica centrada no usuário e que represente as relações entre as entidades presentes na ontologia apresentada. Para isso, propõem a concepção de uma ontologia capaz de refletir as relações interpessoais que acontecem no mundo real, seja entre pessoas ou entre pessoas e recursos.

Mendonça et al. (2014) apresentam um modelo de arquitetura de software que suporta funcionalidades colaborativas e sociais em Ambientes Educacionais Ubíquos. Esta arquitetura promove a extensão e o enriquecimento de uma determinada informação através da definição de mecanismos para sua anotação e classificação, provendo ainda subsídios para sistemas de recomendação e personalização de conteúdo.

Em Park e Kang (2015), é proposto, diante da dificuldade que a maioria dos trabalhos encontram para representar as relações entre os usuários e considerá-los ao mesmo tempo, aumentar as recomendações com base em um gráfico que representa todas as relações explícitas e implícitas em uma rede social. Sugerindo assim uma estrutura que usa os relacionamentos entre os usuários para criar regras de identificação dos vizinhos.

Wang et al. (2015) apresentam uma rede social semântica que se baseia no estilo de vida dos usuários. Desta forma, através dos sensores presentes nos smartphones, esta rede social descobre o estilo de vida de um usuário e mede a semelhança com os estilos dos demais usuários, de modo que recomenda amigos com base no nível de semelhança.

Diante do contexto exposto, este trabalho baseia-se em algumas técnicas mencionadas, porém não se restringe a elas, visto que o mesmo envolve aspectos de recomendação de conteúdos, enriquecimento semântico do perfil do usuário, é desenvolvido para dispositivos móveis, oferecendo a troca de experiências entre os usuários e ainda a possibilidade de acompanhamento de pacientes com doenças crônicas.

\section{MobiLEHealth}

O trabalho aqui apresentando funcionará como uma parte integrante do MobiLEHealth (Mobile Learning Environment for Health) [Mendes Neto et al. 2014; Costa 2015; 
Moreira 2015; Sombra 2015]. O MobiLEHealth é um ambiente de aprendizagem ubíqua no contexto da Saúde 2.0 destinado a pessoas com doenças crônicas. Este ambiente é capaz de adequar-se às características particulares dos usuários, no intuito de disponibilizar conteúdos adequados às suas necessidades. Dessa forma, visa oferecer ao usuário a oportunidade de conhecer mais sobre a sua doença e, assim, obter uma melhoria na sua qualidade de vida. Para conhecimento do paciente, o MobiLEHealth considera o perfil do mesmo e seu contexto atual, além das suas interações com os conteúdos web e mídias sociais [Mendes Neto et al. 2014].

Este ambiente é composto por um conjunto de três sistemas, são eles: Sistema de Recomendação Personalizada de Conteúdos (SRPC); Sistema de Enriquecimento Semântico de Perfil de Usuário (SESPU); e Sistema de Monitoramento Ubíquo de Usuários (SMUU). Esses três sistemas atuam dentro do MobiLEHealth de forma independente, porém de modo integrado.

O SMUU consiste em um ambiente que possui a responsabilidade de realizar o monitoramento ubíquo de atividades cotidianas do usuário [Sombra 2015]. Esse monitoramento deve ser realizado de forma dinâmica, autônoma e transparente. Dessa forma, o ambiente tem a capacidade de capturar e monitorar as interações dos usuários através dos acessos aos conteúdos web e mídias sociais. Como interagem diretamente com o sistema abordado por esse trabalho, o SRPC e o SESPU serão abordados com mais detalhes nas subseções seguintes.

\subsection{Sistema de Recomendação Personalizada de Conteúdo}

Este sistema tem como objetivo apresentar conteúdos relacionados com as características do perfil do usuário, fazendo assim com que a informação proposta seja personalizada e baseada no contexto diário do paciente e na sua condição de saúde.

O SRPC apresenta uma arquitetura baseada em camadas, de forma que cada elemento presente nele seja responsável por técnicas de filtragem e seleção específicas, possibilitando assim a aplicação de variadas técnicas e a seleção cada vez mais eficiente dos conteúdos relevantes ao usuário.

Durante o seu processo, são realizadas inicialmente uma Recomendação Colaborativa (RC) e uma Recomendação Baseada em Conteúdo (RBC). A partir da RC é gerada uma Lista de Colaboradores (LC), que servirá como entrada para a Recomendação Colaborativa Ponderada (RCP). Ao final, a RCP e a RBC geram listas onde os conteúdos presentes nelas são associados e então é realizada a Recomendação Híbrida Ubíqua, que resultará em uma Recomendação Conjunta de itens relacionados com os interesses do usuário.

\subsection{Sistema de Enriquecimento Semântico de Perfil de Usuário}

O objetivo deste sistema é prover meios para determinar os interesses do usuário relacionados à sua saúde, considerando o seu contexto diário. Fazendo assim com que o perfil deste usuário seja enriquecido de forma semântica com base nos domínios de conhecimentos registrados no repositório semântico que ele possui.

O SESPU possui em sua estrutura um analisador de conteúdo, que realiza o processamento de um determinado conteúdo utilizando técnicas de Processamento de 
V Congresso Brasileiro de Informática na Educação (CBIE 2016)

Anais dos Workshops do V Congresso Brasileiro de Informática na Educação (CBIE 2016)

Linguagem Natural, em que busca por termos semelhantes nas ontologias de domínio. Além disso, este sistema possui um enriquecedor semântico, que tem como função gerar anotações semânticas para as correspondências encontradas e as armazenar em uma ontologia de perfil de usuário.

\section{Ambiente Social Virtual Semântico}

A partir dos sistemas presentes no MobiLEHealth, foi desenvolvido um ambiente social virtual com características semânticas que oferece ao paciente com doença crônica conteúdos relevantes de acordo com a sua realidade de vivência com a doença.

Este ambiente coleta as informações de forma semântica do usuário, de modo que, através da ação de um sistema multiagente, os seus traços digitais, pesquisas, amigos e perfil são conhecidos pelo sistema. As informações capturadas por este sistema multiagente são enviadas até o repositório semântico do MobiLEHealth, onde inicialmente é realizada a classificação do paciente de acordo com as suas características de saúde. Essa classificação parte do conhecimento de um profissional de saúde e é realizada através da consulta a uma ontologia de classificação de usuário.

Esta ontologia é uma FOAF e é capaz de inferir relações entre os usuários do ambiente social. Para inferir essas informações, leva-se em consideração o nível de semelhança nos seguintes aspectos: tipo de diabetes; tempo que o paciente possui diabetes; idade do paciente; gênero; afinidade com tecnologia; e quantidade de amigos em comum.

Dessa forma, a ontologia desenvolvida analisa esses pontos e, quando um usuário apresenta informações semelhantes com outro, esses aspectos são pontuados de acordo com a sua relevância e, ao final da análise, há uma pontuação final que determina se a recomendação deve ser feita ou não.

Para realizar as consultas e o acesso a dados desta ontologia é utilizada a SPARQL, uma linguagem voltada para consultas a bases RDF, a qual a FOAF está estruturada.

Após esta classificação, o perfil do paciente será enriquecido com o auxílio do SESPU e, a partir disso, os conteúdos serão recomendados pelo SRPC.

As fontes de tais conteúdos ainda passam pela validação de um profissional de saúde, que as classifica, de forma invisível ao usuário, de acordo com a realidade de vivência que o paciente tem com a doença crônica, garantindo assim que todo conteúdo que é recomendado seja de fato relevante para o paciente. A Figura 1 apresenta a arquitetura de funcionamento deste ambiente, e das ferramentas ligadas a ele, junto com o MobiLEHealth e seus sistemas.

A partir do que é apresentado na Figura 1, é possível visualizar onde o ambiente proposto por esse trabalho e as ferramentas ligadas a ele estão inseridos, bem como o fluxo que as informações seguem após serem coletadas pelo ambiente e enviadas ao repositório de dados. Na Figura 2 são apresentadas algumas telas do ambiente proposto, com (i) o espaço onde são apresentados os conteúdos recomendados, (ii) a sessão onde é possível assistir vídeos que foram selecionados, (iii) o espaço onde aparecem os posts dos amigos e (iv) o local onde é possível gerenciar o perfil do paciente. 
V Congresso Brasileiro de Informática na Educação (CBIE 2016)

Anais dos Workshops do V Congresso Brasileiro de Informática na Educação (CBIE 2016)

Com essas funcionalidades, o paciente tem um ambiente onde consegue acessar informações pertinentes a respeito da sua doença, além de trocar experiências com os demais usuários da rede.

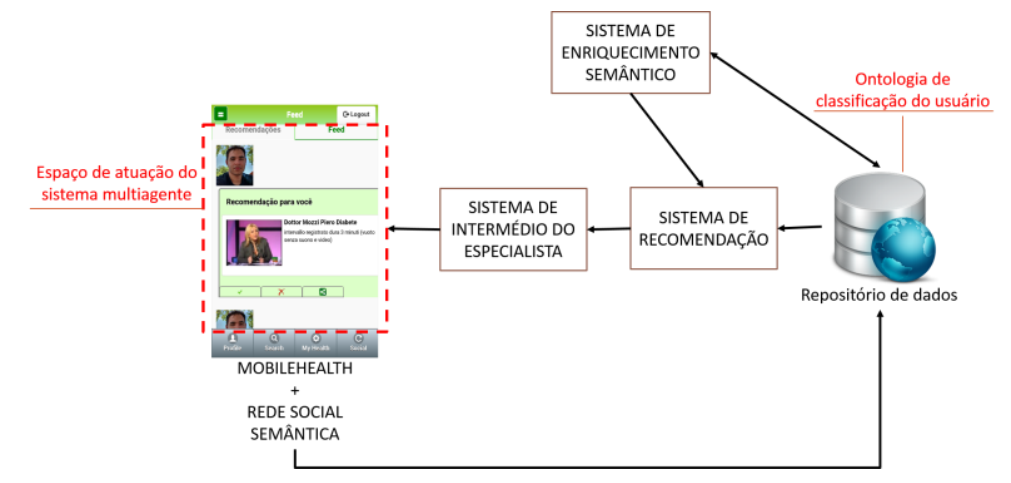

Figura 1 - Arquitetura de funcionamento do sistema proposto.

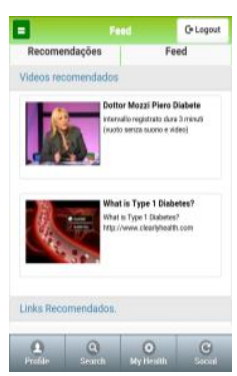

(i)

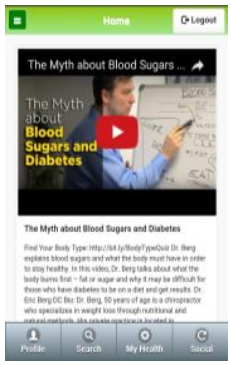

(ii)

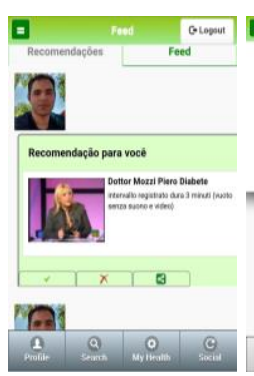

(iii)

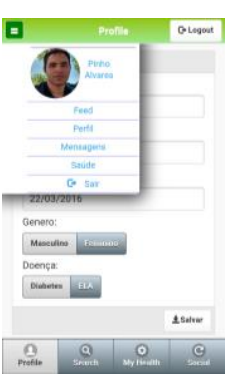

(iv)

Figura 2 - Telas da Rede Social Semântica.

\section{Considerações Finais}

Neste artigo foi apresentado um ambiente social virtual voltado para pacientes com doenças crônicas que, através das ferramentas da Web 2.0, possibilita a aprendizagem informal no âmbito da saúde.

A partir do que foi desenvolvido, é possível perceber que o ambiente abordado apresenta uma alternativa tecnológica para o auxílio na educação a respeito de um problema crônico de saúde. Através dos sistemas que ele possui, bem como daqueles que o MobiLEHealth dispõe, conteúdos personalizados são oferecidos de acordo com a demanda e realidade do paciente, contribuindo assim para que a aprendizagem informal ocorra e possibilite a evolução do conhecimento do diabético a respeito da sua doença e do tratamento inerente a ela. Como trabalho futuro têm-se a validação de todo o sistema através de usuários reais.

\section{Referências}

Castleton, G.; Gerber, R.; Pillay, H. (Org.). Improving Workplace Learning:Emerging International Perspectives. New York: Nova Science Publishers Inc, 2006.

Chenu-Abente, R.; Giunchiglia, F.; Cernuzzi, L. Towards semantic social networks. 2015 Latin American Computing Conference (clei), [s.1.], p.10-23, out. 2015. Institute of Electrical \& Electronics Engineers (IEEE). DOI: 10.1109/clei.2015.7359998. 
V Congresso Brasileiro de Informática na Educação (CBIE 2016)

Anais dos Workshops do V Congresso Brasileiro de Informática na Educação (CBIE 2016)

Costa, A. A. L. Mecanismo de Recomendação Personalizada de Conteúdos para apoiar um Ambiente de Aprendizagem Informal no contexto da Saúde. 2015. $92 \mathrm{f}$. Dissertação (Mestrado) - Curso de Pós-graduação em Ciência da Computação, Universidade Federal Rural do Semi-Árido e Universidade do Estado do Rio Grande do Norte, Mossoró, 2015.

Fernandez-Luque, L. et al. Personalized health applications in the Web 2.0: the emergence of a new approach Annual International Conference of the IEEE Engineering in Medicine and Biology Society (EMBC). Anais... Buenos Aires, Argentina: $\quad 2010 . \quad$ Disponível em: $<$ http://ieeexplore.ieee.org/ielx5/5608545/5625939/05628087.pdf?tp=\&arnumber $=56$ 28087\&isnumber $=5625939>$

Fioravanti, A. et al. A mobile feedback system for integrated e-health platforms to improve self-care and compliance of diabetes mellitus patients. In: Engineering in Medicine and Biology Society,EMBC, 2011 Annual International Conference of the IEEE. [S.1.: s.n.], 2011. p. 3550-3553. ISSN 1557-170X.

IBGE. Indicadores Sociodemográficos e de Saúde no Brasil. Pesquisa, no 25. Rio de Janeiro: Instituto Brasileiro de Geografia e Estatística, 2009. Disponível em: $<$ http://www.ibge.gov.br/home/estatistica/populacao/indic_sociosaude/2009/indicsau de.pdf $>$.

Jacopetti, A. M. Práticas sociais e de comunicação de pacientes renais no Facebook da Fundação Pró-Rim. Revista de Estudos da Comunicação, Curitiba, v. 12, n. 27, p.8189, abr. 2011. Quadrimestral.

Jiugen, Y.; Ruonan, X.; Xiaoqiang, H. Constructing informal learning mode based on social software. In: 2011 6TH INTERNATIONAL CONFERENCE ON COMPUTER SCIENCE EDUCATION (ICCSE), ago. 2011, [S.1: s.n.], ago. 2011. p. $1227-1230$.

Kinsella, S. et al. Applications of Semantic Web Methodologies and Techniques to Social Networks and Social Websites. Reasoning Web, [s.1.], p.171-199, 2008. Springer Science + Business Media. DOI: 10.1007/978-3-540-85658-0_5.

Lee, K. et al. Building a Semantic Social Network Based on Interpersonal Relationships. 2012 Third Ftra International Conference On Mobile, Ubiquitous, And Intelligent Computing, [s.1.], p.90-95, jun. 2012. Institute of Electrical \& Electronics Engineers (IEEE). DOI: 10.1109/music.2012.23.

Mendes Neto, F. M. et al. An Approach for Recommending Personalized Contents for Homecare Users in the Context of Health 2.0. EATIS '14, 2014, New York, NY, USA. Anais... New York, NY, USA: ACM, 2014. p. 33:1-33:2.

Mendonça, I. E. S. et al. Explorando Funcionalidades Sociais e Colaborativas em Ambientes Educacionais Ubíquos. In: SIMPÓSIO BRASILEIRO DE INFORMÁTICA NA EDUCAÇÃO, 25., 2014, Dourados. Anais... . Dourados: Cbie, 2014. p. 486 - 495.

Montiel, E. M. S. et al. Sistema de Educacão em Saúde no Tratamento do Diabetes Mellitus Tipo 2. In: SIMPÓSIO BRASILEIRO DE INFORMÁTICA NA EDUCAÇÃO, 26., 2015, Maceió. Anais... . Maceió: Sbie, 2015. p. 328 - 337. 
V Congresso Brasileiro de Informática na Educação (CBIE 2016)

Anais dos Workshops do V Congresso Brasileiro de Informática na Educação (CBIE 2016)

Moreira, J. D. C. Enriquecimento semântico de perfil de usuário para apoio a um modelo de aprendizagem informal no contexto da saúde. 2015. 92 f. Dissertação (Mestrado) - Curso de Pós-graduação em Ciência da Computação, Universidade Federal Rural do Semi-Árido e Universidade do Estado do Rio Grande do Norte, Mossoró, 2015.

Pankong, N.; Prakancharoen, S.; Buranarach, M. A combined semantic social network analysis framework to integrate social media data. Knowledge And Smart Technology (kst), [s.1.], p.10-16, jul. 2012. Institute of Electrical \& Electronics Engineers (IEEE). DOI: 10.1109/kst.2012.6287736.

PARK, Sangun; KANG, Juyoung. Recommendation using analysis of semantic social network in social network services.Proceedings Of The 17th International Conference On Electronic Commerce 2015 - Icec '15, [s.1.], p.10-15, 2015. Association for Computing Machinery (ACM). http://dx.doi.org/10.1145/2781562.2781603.

Paydar, S.; Kahani, M.; Zarrinkalam, F. PAD: A semantic social network. Iccke 2013, [s.1.], p.6-12, out. 2013. Institute of Electrical \& Electronics Engineers (IEEE). DOI: 10.1109/iccke.2013.6682853.

Portal Brasil (Org.). Pesquisa revela que 57,4 milhões de brasileiros têm doença crônica. 2014. Disponível em: <http://www.brasil.gov.br/saude/2014/12/pesquisa-revela-que57-4-milhoes-de-brasileiros-tem-doenca-cronica>. Acesso em: 1 mai. 2016.

Santos, F. R. L. Web 2.0 e Saúde 2.0:: Como entender o mundo em que vivemos hoje? - Parte 2. 2008. Disponível em: <http://www.patologiadaatm.com.br/web-20-esaude-20-como-entender-o-mundo-em-que-vivemos-hoje-parte-2/>. Acesso em: 13 jun. 2015.

Sombra, E. L. Mobilehealth: um ambiente de apoio à saúde 2.0. 2015. $101 \mathrm{f}$. Dissertação (Mestrado) - Curso de Pós-graduação em Ciência da Computação, Universidade Federal Rural do Semi-Árido e Universidade do Estado do Rio Grande do Norte, Mossoró, 2015.

Wang, M.; Shen, R. Message design for mobile learning: Learning theories, human cognition and design principles. British Journal of Educational Technology, v. 43, n.4, p.561-575, 1 jul. 2012.

Wang, Zhibo et al. Friendbook: A Semantic-Based Friend Recommendation System for Social Networks. Ieee Transactions On Mobile Computing, [s.1.], v. 14, n. 3, p.538551, 1 mar. 2015. Institute of Electrical \& Electronics Engineers (IEEE). http://dx.doi.org/10.1109/tmc.2014.2322373.

Weitzel, L.; Oliveira, J. P. M. Sistemas de recomendação de informação em saúde baseado no perfil do usuário. Journal Of Health Informatics. São Paulo, p. 1-7. set. 2010 .

WHO. Health Promotion Glossary. . Geneva, Switzerland: World Health Organization, $2008 . \quad$ Disponível em: $<$ http://www.who.int/healthpromotion/about/HPR\%20Glossary\%201998.pdf?ua=1>. 